\title{
Feed-Forward Neural Network Soft-Sensor Modeling of Flotation Process Based on Particle Swarm Optimization and Gravitational Search Algorithm
}

\author{
Jie-Sheng Wang and Shuang Han \\ School of Electronic and Information Engineering, University of Science and Technology Liaoning, Anshan, Liaoning 114044, China \\ Correspondence should be addressed to Jie-Sheng Wang; wang_jiesheng@126.com
}

Received 18 March 2015; Accepted 25 May 2015

Academic Editor: Adel Elmaghraby

Copyright (C) 2015 J.-S. Wang and S. Han. This is an open access article distributed under the Creative Commons Attribution License, which permits unrestricted use, distribution, and reproduction in any medium, provided the original work is properly cited.

\begin{abstract}
For predicting the key technology indicators (concentrate grade and tailings recovery rate) of flotation process, a feed-forward neural network (FNN) based soft-sensor model optimized by the hybrid algorithm combining particle swarm optimization (PSO) algorithm and gravitational search algorithm (GSA) is proposed. Although GSA has better optimization capability, it has slow convergence velocity and is easy to fall into local optimum. So in this paper, the velocity vector and position vector of GSA are adjusted by PSO algorithm in order to improve its convergence speed and prediction accuracy. Finally, the proposed hybrid algorithm is adopted to optimize the parameters of FNN soft-sensor model. Simulation results show that the model has better generalization and prediction accuracy for the concentrate grade and tailings recovery rate to meet the online soft-sensor requirements of the real-time control in the flotation process.
\end{abstract}

\section{Introduction}

Flotation is known as froth flotation, and it is a physicochemical reaction process. Flotation is the process which is based on the differences of the surface property of solid materials to separate useful minerals and gangue by means of the buoyancy of air bubbles from ore pulp by this method to improve the concentrate grade [1]. In the production process of flotation, concentrate grade and other economic and technical indicators are key control indicators of the production process. Process control indicators of domestic flotation process are mainly based on an experienced operator to observe the information (such as foam color, size, flow rate, and texture features) which is provided by the bubble state formed on the surface of the flotation tank and to adjust the flotation level and change agents system $[2,3]$. Inference estimate (soft-sensor) technology can effectively solve the online estimation problems where the flotation process is difficult to online measure the economic and technical indicators.
Domestic and foreign scholars carry through the research on soft-sensor modeling of the key technical indicators in the flotation process and make a lot of achievements [4-16]. Hargrave and Hall study the diagnosis and analysis methods of the metal grade, quality, and flow rate in flotation process by using the color and surface tissue [4]. Bartolacci et al. use multivariate image analysis (MIA) and partial least squares (PLS) methods to establish the experience prediction model of flotation grade [5]. Morar et al. utilize the machine vision method to predict the performance of the flotation process, such as concentrate grade and tailings recovery rate [6]. Moolman and many other scholars created a bubble dynamic model based on image processing through researching flotation foam structure and calculated the content of useful minerals in foam through this model [7].

At home, Yang et al. put forward a bubble image segmentation method based on the clustering presplit and the accuracy distance reconstruction [8]. In that the softsensor method with multiple models can improve the overall prediction accuracy and have the characteristic of robustness; 


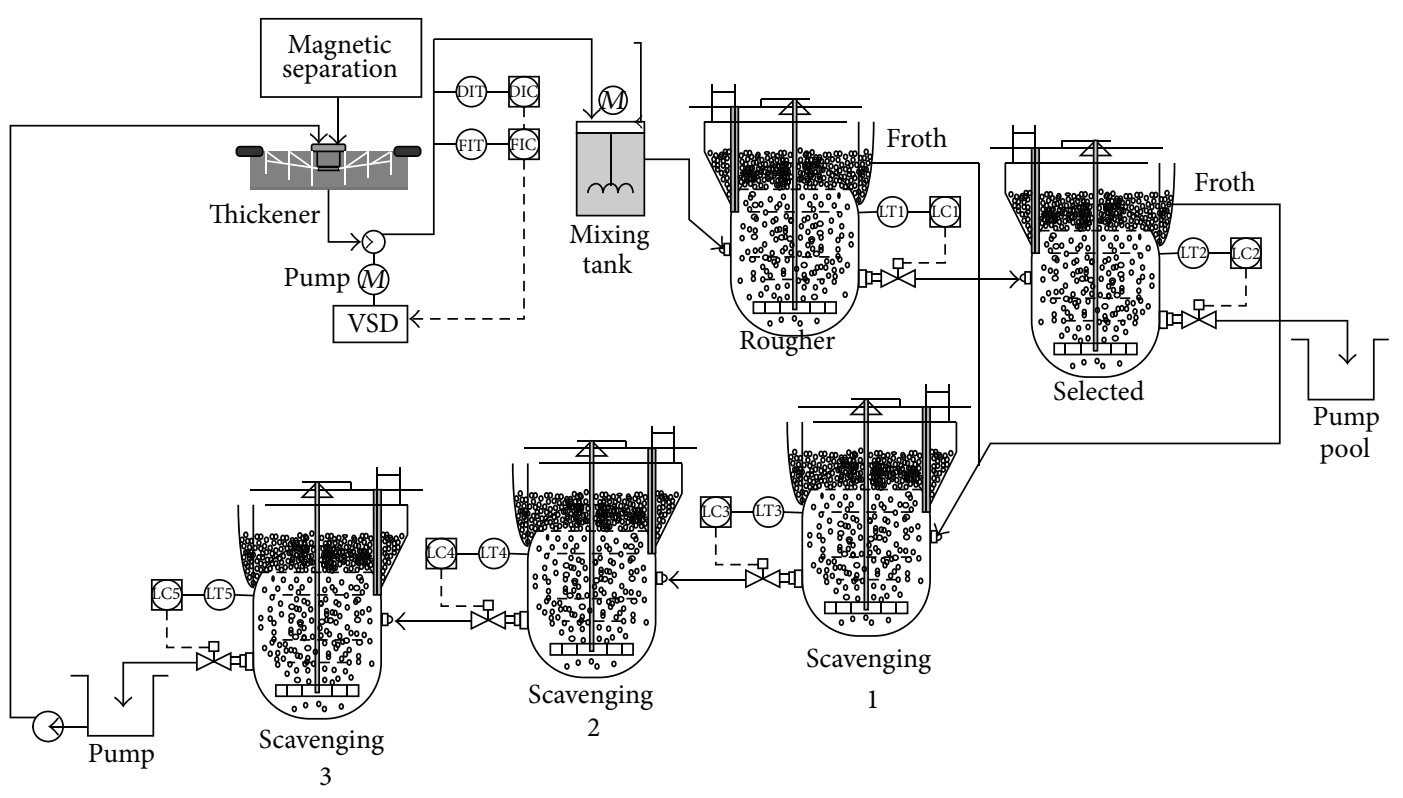

FIGURE 1: Technique flowchart of flotation process.

Wang et al. present a multi-T-S fuzzy neural network softsensor model of flotation process based on the FCM clustering algorithm [9]. Yang et al. use the flotation froth video image features as auxiliary variables and establish a softsensor model of the flotation pulp $\mathrm{pH}$ value based on the sparse polynuclear least squares support vector machine (SVM) and use Schmidt orthogonalization theory to reduce the multinuclear matrix [10]. Li et al. set up a soft-sensor mode by combining the principal component analysis (PCA) and extreme learning machine (ELM) methods [11]. Zhou et al. extracted color and size characteristics of the foam by using digital image processing method and established a recovery prediction model [12]. Wang and Zhang proposed a kind of soft-sensor model of economic and technical index based on PCA and ANFIS and combining PSO algorithm with LSM put forward a new learning process to optimize parameters of ANFIS [13]. Geng and Chai utilized least squares support vector machine to establish soft-sensor model of concentrate grade and tailing grade in the flotation process based on analyzing related influencing factors of concentrate grade and tailing grade of the flotation process technology indicators [14]. Wang et al. proposed the features extraction method of flotation froth images and BP neural network soft-sensor model of concentrate grade optimized by shuffled cuckoo searching algorithm [15]. Wang et al. proposed an echo state network (ESN) based fusion softsensor model optimized by the improved glowworm swarm optimization (GSO) algorithm. Simulation results show that the model has better generalization and prediction accuracy [16].

This paper proposes a feed-forward neural network (FNN) soft-sensor model by using process datum in the flotation process for predicting the flotation concentrate grade and recovery rate, which is optimized by the PSOGSA algorithm. Simulation results verify the validity of the proposed soft-sensor model. The paper is organized as follows. In Section 2, the technique flowchart of flotation process is introduced. The FNN soft-sensor model of flotation process optimized by PSO-GSA algorithm is presented in Section 3. In Section 4, experiment and simulation results are introduced in detail. Finally, the conclusion illustrates the last part.

\section{Technique Flowchart of Flotation Process}

Flotation process is used to separate useful minerals and gangue based on the differences of the surface property of solid materials. Figure 1 is a typical iron ore flotation process consisting of the roughing, concentration, and scavenging [11]. The system input is the fine concentrate pulp which is early output of beneficiation process in the forepart. The pulp density is about $38 \%$ and concentrate grade is about $64 \%$. Inlet pulp is fed into the high-stirred tank through the pulp pipeline by feed pump. At the same time, the flotation reagent according to a certain concentration ratio is also fed into high-stirred tank through dosing pump. On the other hand, the pulp temperature must reach a suitable flotation temperature by heating. If the dosage is appropriate, the flotation cells can output a grade of $68.5 \%-69.5 \%$ concentrate $[15,16]$.

The control objective of flotation process is to ensure the concentrate grade and the tailings recovery rate are within a certain target range. In common, based on the offline artificial laboratory to get grade values, the operators adjust the flotation cell level and the amount of flotation reagent addition. Due to the artificial laboratory for two hours at a time, when the process variables and boundary conditions change in the flotation process, they cannot timely adjust the flotation operation variables, which results in such phenomena that the flotation concentrate grade and the tailings recovery rate are too high or too low $[15,16]$. 
TABLE 1: Soft-sensor modeling data of flotation process.

\begin{tabular}{|c|c|c|c|c|c|c|c|}
\hline \multirow[b]{2}{*}{ Number } & \multicolumn{5}{|c|}{ Auxiliary variables } & \multicolumn{2}{|c|}{ Dominant variables } \\
\hline & $\begin{array}{c}\text { Feed } \\
\text { concentration } \\
(\%)\end{array}$ & Feed flow $\left(\mathrm{m}^{3} / \mathrm{h}\right)$ & Feed grade (\%) & $\begin{array}{c}\text { Feed granularity } \\
(\%)\end{array}$ & $\begin{array}{l}\text { Medicament flow } \\
(\mathrm{L} / \mathrm{min})\end{array}$ & $\begin{array}{l}\text { Concentrate } \\
\text { grade }(\%)\end{array}$ & $\begin{array}{c}\text { Recovery rate } \\
(\%)\end{array}$ \\
\hline 1 & 62.76 & 329 & 35 & 90 & 15.5 & 70.51 & 97.7 \\
\hline 2 & 63.67 & 297 & 35 & 90 & 11.5 & 69.74 & 97.2 \\
\hline 3 & 65.07 & 285 & 37 & 92 & 11.3 & 69.69 & 97.0 \\
\hline 4 & 65.48 & 214 & 36 & 95 & 7.5 & 68.98 & 93.5 \\
\hline$\vdots$ & $\vdots$ & $\vdots$ & $\vdots$ & $\vdots$ & $\vdots$ & $\vdots$ & $\vdots$ \\
\hline 600 & 65.9 & 310 & 36 & 96 & 5.5 & 67.29 & 90.2 \\
\hline
\end{tabular}

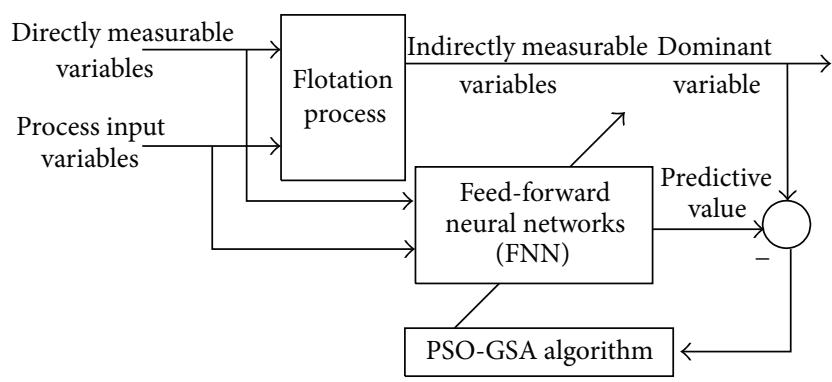

FIgURE 2: Structure of the proposed soft-sensor model of flotation process.

By analyzing the flotation technique, the process variables and boundary conditions mainly include feed grade $x_{1}$, feed flow rate $x_{2}$, feed concentration $x_{3}$, feed granularity $x_{4}$, and medicament flow rate $x_{5}$. The modeling data is shown in Table 1.

\section{Soft-Sensor Modeling of Flotation Process}

3.1. Structure of Soft-Sensor Model. The structure of the proposed feed-forward neural network (FNN) soft-sensor model of the flotation process based on PSO-GSA algorithm is shown in Figure 2.

The auxiliary variables of soft-sensor model proposed in this paper are feed grade, feed concentration, feed flow, feed granularity, and medicament flow. Then the samples composed of the auxiliary variable are normalized as the model input. Finally, parameters of FNN soft-sensor model are optimized by PSO-GSA algorithm. Thereby, the accurate prediction of concentrate grade and tailing recovery rate of the flotation process is achieved. Considering a multiinput single-output (MISO) system, the training set can be represented as $D=\left\{Y, X_{i} \mid i=1,2, \ldots, m\right\}$, where $Y$ represents the output and $X_{i}$ denotes the $i$ th input vector and it can be represented as $X_{i}=\left[x_{1 i}, x_{2 i}, \ldots, x_{n i}\right]^{\prime}(n$ is the number of the training samples and $m$ is the number of the input variables). The establishment of soft-sensor model needs a data set from the normal working condition as the modeling data. Assuming the $m$ process variables, $n$ data vector samples comprise the test data matrix $X \in R^{n \times m}$. In

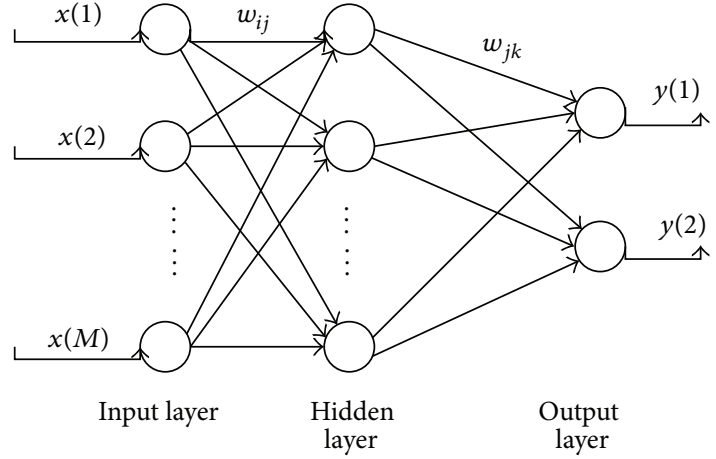

FIGURE 3: Structure of feed-forward neural network (FNN).

order to avoid the effect of different dimensions of process variables for results and be convenient for mathematical handling, it is necessary to normalize the data. Assume the mean vector of $X$ is $\mu$ and the standard deviation vector is $\sigma$. The process variables after the normalization are described as follows:

$$
\widehat{X}=\frac{(X-\mu)}{\sigma} .
$$

Then the input vectors $\widehat{X}$ of the training samples are fed into FNN soft-sensor model to obtain the predictive output $\widehat{Y}$. On the other hand, the root mean square error (RMSE) is adopted as the fitness value of soft-sensor model:

$$
\text { RMSE }=\sqrt{\sum_{k=1}^{n} \frac{\left(\widehat{Y}_{k}-Y_{k}^{*}\right)^{2}}{n}},
$$

where $Y^{*}$ is the actual output of the training samples.

3.2. Feed-Forward Neural Network (FNN). In accordance with different layers of the feed-forward neural network (FNN), it can be divided into single-layer feed-forward neural network and multilayer feed-forward neural network. The multilayer FNN is adopted in this paper, which includes an input layer, a hidden layer, and an output layer, whose structure is shown in Figure 3. 
Assume that the input layer has $M$ inputs; $i(i=$ $1,2, \ldots, M)$ represents any input. The hidden layer has $N$ inputs; $j(j=1,2, \ldots, N)$ represents any inputs. The connection weight values between the input layer and the hidden layer are $w_{i j}(i=1,2, \ldots, M ; j=1,2, \ldots, N)$. The connection weight values between the hidden layer and the output layer are $w_{j k}(k=1,2)$. Assume that the input of the hidden layer neurons is $u_{j}$; output is $v_{j}$. Input of output layer neurons is $o_{k}$; total output is $y_{k}$. So the calculation of FNN can be represented as follows:

$$
\begin{aligned}
& u_{j}=\sum_{i=1}^{M} w_{i j} x(i), \\
& v_{j}=f\left[u_{j}\right]=f\left[\sum_{i=1}^{M} w_{i j} x(i)\right], \\
& o_{k}=\sum_{j=1}^{N} w_{j k} v_{j}, \\
& y_{k}=f\left[o_{k}\right]=f\left[\sum_{j=1}^{N} w_{j k} v_{j}\right],
\end{aligned}
$$

where $f()$ represents the transfer function between input and output of the hidden layer and the output layer, which is also called the activation function.

\subsection{FNN Soft-Sensor Model Optimized by Hybrid PSO-GSA Algorithm}

3.3.1. Particle Swarm Optimization (PSO) Algorithm. Particle swarm optimization (PSO) algorithm is a kind of swarm intelligent optimization algorithm, which is inspired by the birds' migration and swarming behavior during the foraging process. Due to its simplicity, it has been widely used in many optimization problems. It uses a large number of particles (potential solutions) to find the best solution in the search space, where each particle corresponds to a fitness value, and the velocity of the particles is decided by their flight direction and distance. Adjust the individual best value and the global best value to meet the requirements dynamically $[17,18]$.

Assume that, in an $S$-dimensional searching space, $n$ particles consist of the population $X=\left(x_{1}, x_{2}, \ldots, x_{n}\right)$, where the $i$ th particle is expressed as an $S$-dimensional vector $x_{i}=\left(x_{i 1}, x_{i 2}, \ldots, x_{i s}\right)^{T}$. It represents the position of the $i$ th particle in the searching space (the potential solutions of the discussed problem). $v_{i}=\left(v_{i 1}, v_{i 2}, \ldots, v_{i s}\right)^{T}$ represents the velocity of the $i$ th particle, pbest ${ }_{i}=\left(p_{i 1}, p_{i 2}, \ldots, p_{i s}\right)^{T}$ represents the individual best value, and gbest $=\left(g_{1}, g_{2}, \ldots, g_{s}\right)^{T}$ represents the global best value. According to the following equations, the position and velocity vector of the particles are updated:

$$
\begin{aligned}
v_{i}^{t+1}= & w v_{i}^{t}+c_{1} \times \text { rand } \times\left(\text { pbest }_{i}-x_{i}^{t}\right)+c_{2} \times \text { rand } \\
& \times\left(\text { gbest }-x_{i}^{t}\right), \\
x_{i}^{t+1}= & x_{i}^{t}+v_{i}^{t+1},
\end{aligned}
$$

where $w$ is the inertia weight, $t$ is the number of iterations, $v_{i}^{t}$ represents the velocity of $i$ th particle in $t$ iteration, $c_{1}$ is the particle's acceleration weighting coefficient, $c_{2}$ is a global acceleration weighting coefficient, $c_{1}$ and $c_{2}$ are learning factors (usually $c_{1}=c_{2}=2$ ), rand is a random number between 0 and 1 , and $x_{i}^{t}$ represents the current location of the $i$ th particle in $t$ th iteration.

The first part $w v$ of (4) represents the search capability of PSO algorithm, and the second part $c_{1} \times$ rand $\times\left(\right.$ pbest $\left._{i}-x_{i}^{t}\right)$ and the third part $c_{2} \times \operatorname{rand} \times\left(\right.$ gbest $\left.-x_{i}^{t}\right)$ represent individual and global optimization ability of particles. In the searching space, the position vector of the particles is randomly generated. In each iteration, (4) is used to update the velocity vector of particles. After determining the velocity of the particles, the particle position vector is updated by (5). The position vector of the particles will be constantly changed until the termination condition is satisfied.

3.3.2. Gravitational Search Algorithm (GSA). In 2009, Rashedi et al. put forward a gravitational search algorithm (GSA), which is a heuristic optimization algorithm [19]. It uses the physics law to find the optimal solution in the searching space. Inspiration of the GSA comes from Newton's universal gravitation law. Gravity is a force of attraction that exists between any two masses, any two bodies, or any two particles. The size of the gravitational force is proportional to their product of the quality and inversely proportional to the distance between them. In this algorithm, each individual represents a potential solution, each of the potential solutions corresponds to a fitness value, and the fitness value is represented by the quality of the individual. Everything with massive particle in the universe attracts all other massive particles; a large mass of individuals is subject to greater gravitation. So a large mass of individuals is near to the global best value. The flowchart of gravitation search algorithm is described as in Figure 4.

Suppose, in an $n$-dimensional searching space, $N$ substances constitute a population. Each individual's position (potential solution) is defined as follows:

$$
x_{i}=\left(x_{i}^{1}, \ldots, x_{i}^{d}, \ldots, x_{i}^{n}\right), \quad i=1,2, \ldots, N,
$$

where $x_{i}^{d}$ is the position of substance $i$ in $d$-dimension of the space.

In the searching space, all individuals are randomly placed in the $t$ th generation. So the gravity of substance $j$ attracting substance $i$ in $d$-dimensional space is defined as

$$
F_{i j}^{d}(t)=G(t) \frac{M_{p i}(t) \times M_{a j}(t)}{R_{i j}(t)+\varepsilon}\left(x_{j}^{d}(t)-x_{i}^{d}(t)\right),
$$




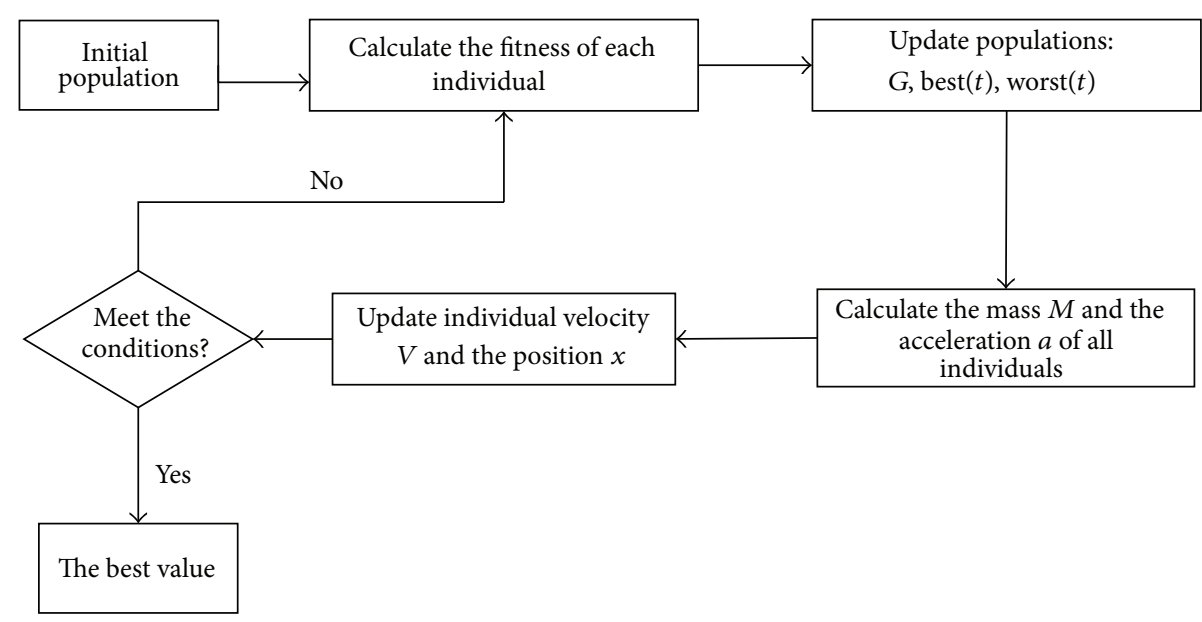

FIGURE 4: Flowchart of gravitation search algorithm.

where $M_{a j}$ represents the active gravitational mass of individual $j, M_{p i}$ represents the passive gravitational mass of individual $i, G(t)$ represents the gravitational constant in $t$ th generation, $\varepsilon$ is a small constant, and $R_{i j}(t)$ represents the Euclidean distance between substance $i$ and substance $j$.

The gravitational constant $G$ and the Euclidean distance between substance $i$ and substance $j$ are calculated as follows:

$$
\begin{aligned}
G(t) & =G_{0} \times \exp \left(-\alpha \times \frac{\text { iter }}{\text { maxiter }}\right), \\
R_{i j} & =\left\|x_{i}(t), x_{j}(t)\right\|_{2},
\end{aligned}
$$

where $\alpha$ is the decreasing coefficient (constant), $G_{0}$ represents the initial gravitational constant, iter represents the number of current iterations, and maxiter represents the number of maximum iterations.

In the $d$-dimensional searching space, all gravity which acts on the material $i$ is calculated as follows:

$$
F_{i}^{d}(t)=\sum_{j=1, j \neq i}^{N} \operatorname{rand}_{j} F_{i j}^{d}(t),
$$

where rand $_{j}$ is a random number between 0 and 1 .

According to Newton's motion law, the acceleration of the material $i$ is proportional to force in $d$ dimension and inversely proportional to the inverse of the mass. The acceleration of substance is calculated as follows:

$$
a_{i}^{d}(t)=\frac{F_{i}^{d}(t)}{M_{i i}(t)^{\prime}},
$$

where $t$ represents the number of current iterations and $M_{i}$ represents the mass of substance $i$. Speed and position of substance $i$ are updated by the following equations:

$$
\begin{aligned}
& v_{i}^{d}(t+1)=\operatorname{rand}_{i} \times v_{i}^{d}(t)+a_{i}^{d}(t), \\
& x_{i}^{d}(t+1)=x_{i}^{d}(t)+v_{i}^{d}(t+1),
\end{aligned}
$$

where rand $_{i}$ is a random number between 0 and 1 .
It can be seen, from the above two equations, that the current speed of a substance is defined as the part of the final speed $\left(0 \leq \operatorname{rand}_{i} \leq 1\right)$ and its acceleration. The current position of substance is equal to its final velocity and the current position. The fitness represents the quality of the material, which means that the greater the quality of the material, the higher the efficiency. According to the above formula, the heavier the material, the greater the gravity and the slower the movement. The quality of materials is updated by using the following equation:

$$
m_{i}(t)=\frac{\text { fit }_{i}(t)-\operatorname{worst}(t)}{\operatorname{best}(t)-\operatorname{worst}(t)},
$$

where $\mathrm{fit}_{i}(t)$ represents the fitness value of substance $i$ in $t$ th generation, best $(t)$ represents the best individual fitness value in $t$ th generation, and $\operatorname{worst}(t)$ represents the worst individual fitness value. With regard to the minimization problem, best $(t)$ and worst $(t)$ are calculated as follows:

$$
\begin{aligned}
\operatorname{best}(t) & =\min _{j \in\{1, \ldots, N\}} \text { fit }_{j}(t), \\
\text { worst }(t) & =\max _{j \in\{1, \ldots, N\}} \text { fit }_{j}(t) .
\end{aligned}
$$

The standardization of quality is defined by the following formula:

$$
M_{i}(t)=\frac{m_{i}(t)}{\sum_{j=1}^{N} m_{j}(t)} .
$$

3.3.3. PSO-GSA Algorithm. Although GSA has better optimization capability, the material appeared to have low convergence in the process of moving to the optimal value and to be easy to fall into local optimum phenomenon. So PSO algorithm is used to update the position and velocity of the individual in order to make up this shortcoming of GSA. The basic idea of PSO-GSA is described as follows. Firstly, generate the initial position vector $X=\left(x_{1}, x_{2}, \ldots, x_{n}\right)$ and velocity vector $v_{i}=\left(v_{i 1}, v_{i 2}, \ldots, v_{i s}\right)^{T}$ of $N$ individuals 
randomly. According to the initial positions of all individuals, calculate the fitness value corresponding to each individual and record the best fitness value best $(t)$ and the worst fitness value worst $(t)$ and the corresponding position vector of the individuals. The quality of individuals is calculated by (13)(15). Then calculate the gravitational constant $G$ and the Euclidean distance between two individuals and the individual's gravitation $F$ and acceleration $a$. At this time, according to (11)-(12), the global search ability of PSO algorithm is used to update velocity and position of the individual, and then the fitness value and the corresponding optimal value are calculated. The best individual is obtained until the number of iterations is reached. Consider

$$
\begin{aligned}
v_{i}(t+1)= & w \times v_{i}(t)+c_{1}^{\prime} \times \operatorname{rand} \times a c_{i}(t)+c_{2}^{\prime} \times \text { rand } \\
& \times\left(\text { gbest }-x_{i}(t)\right),
\end{aligned}
$$

where $v_{i}(t)$ represents the velocity of the material $i$ in $t$ th generation, $c_{j}^{\prime}$ is an acceleration coefficient, $w$ is the inertia weight, rand represents a random number between 0 and $1, a c_{i}(t)$ indicates the acceleration of substance $i$ in $t$ th generation, and gbest represents the optimal solution so far.

After updating the velocity vector, the location vector of substance is updated based on the following equation:

$$
x_{i}(t+1)=x_{i}(t)+v_{i}(t+1) .
$$

3.3.4. Procedure of PSO-GSA Algorithm Optimizing FNN. In this paper, the PSO-GSA hybrid algorithm is applied to optimize the parameters of the FNN soft-sensor model, whose aim is to improve the convergence speed and predictive accuracy. PSO-GSA algorithm is different from GSA. It adopts PSO algorithm to update its velocity and position, until it reaches the number of iterations or accuracy. Because the prediction accuracy of FNN soft-sensor model is related to the initial connection weights and thresholds, if the parameters are improper, it will lead to decline of prediction accuracy. Therefore, the hybrid PSO-GSA algorithm is used to optimize FNN soft-sensor model. The flowchart of PSOGSA algorithm optimizing FNN is shown in Figure 5.

The algorithm procedure is described as follows in detail.

Step 1 (initialize parameters). Determine the topology of FNN. Initialize the weights $w$ and the threshold value $b$ of FNN and predispose the training samples of flotation process. Initialize the population size $N$ and the number of iterations $t$.

Step 2 (generate the population randomly). Set the initial position $X=\left(x_{1}, x_{2}, \ldots, x_{n}\right)$ and initial velocity $v_{i}=\left(v_{i 1}, v_{i 2}, \ldots, v_{i s}\right)^{T}$, the learning factors $c_{1}$ and $c_{2}$, and the inertia weight $w$. Initialize the global best value gbest and individual best value pbest, the decreasing coefficient $\alpha$, and the gravitational constant $G_{0}$.

Step 3. The position of each individual corresponds to a set of weights and thresholds of FNN soft-sensor model. Train FNN and calculate the fitness value fit $(t)$, of each individual. Find the optimal fitness value best $(t)$ and the worst fitness value worst $(t)$ and record the best position gbest.

Step 4. According to (13)-(15), calculate the mass of the individual, the gravitational constant $G$, and the Euclidean distance between two individuals. Then calculate the gravitation $F$ and the acceleration $a$ of the individual. At this time, according to (11)-(12), the global search ability of PSO algorithm is adopted to update the velocity and position of the individuals. Then calculate the corresponding fitness value fit $(t)$ and the optimal value gbest' of the individual. The optimal value gbest ${ }^{\prime}$ is compared with the optimal value gbest in Step 3; the optimal individual after comparison is recorded as gbest".

Step 5. Determine whether the termination condition is reached or not (the objective function reaches a certain value or the number of iterations reaches the maximum). If the termination condition is not met, the procedure returns to Step 3.

Step 6 (model validation). The corresponding parameters of the best individual gbest are set as the weights and thresholds of FNN soft-sensor model and verify the established FNN soft-sensor model with the testing data.

\section{Simulation Results}

In this paper, 600 pieces of input data are selected as input and the concentrate grade and recovery rate are output of FNN soft-sensor model for the flotation process, where 550 samples are training data and the remaining 50 samples are testing data. Finally, the weights and thresholds of FNN are optimized by PSO-GSA algorithm. This paper selects the following five performance indexes to verify the prediction accuracy of different soft-sensor models, whose calculation equations are described as follows:

$$
\begin{aligned}
\text { NRMSE } & =\sqrt{\frac{1}{T\left\|y_{d}\right\|^{2}} \sum_{t=1}^{T}\left(y(t)-y_{d}(t)\right)^{2},} \\
\text { MSE } & =\frac{1}{T} \sum_{t=1}^{T}\left(y(t)-y_{d}(t)\right)^{2}, \\
\text { MAPE } & =\frac{100}{T} \sum_{t=1}^{T} \frac{\left|y(t)-y_{d}(t)\right|}{y_{d}(t)}, \\
\text { RMSE } & =\left[\frac{1}{T} \sum_{t=1}^{n}\left(y_{d}(t)-y(t)\right)^{2}\right]^{1 / 2}, \\
\text { SSE } & =\sum_{t=1}^{n}\left(y_{d}(t)-y(t)\right)^{2},
\end{aligned}
$$

where $T$ is the number of the prediction samples, $y(t)$ is the predicted value, and $y_{d}(t)$ is the actual value.

The input dimension of FNN is 5 , the number of neurons is 20 in the hidden layer, and the output dimension is 2 . 


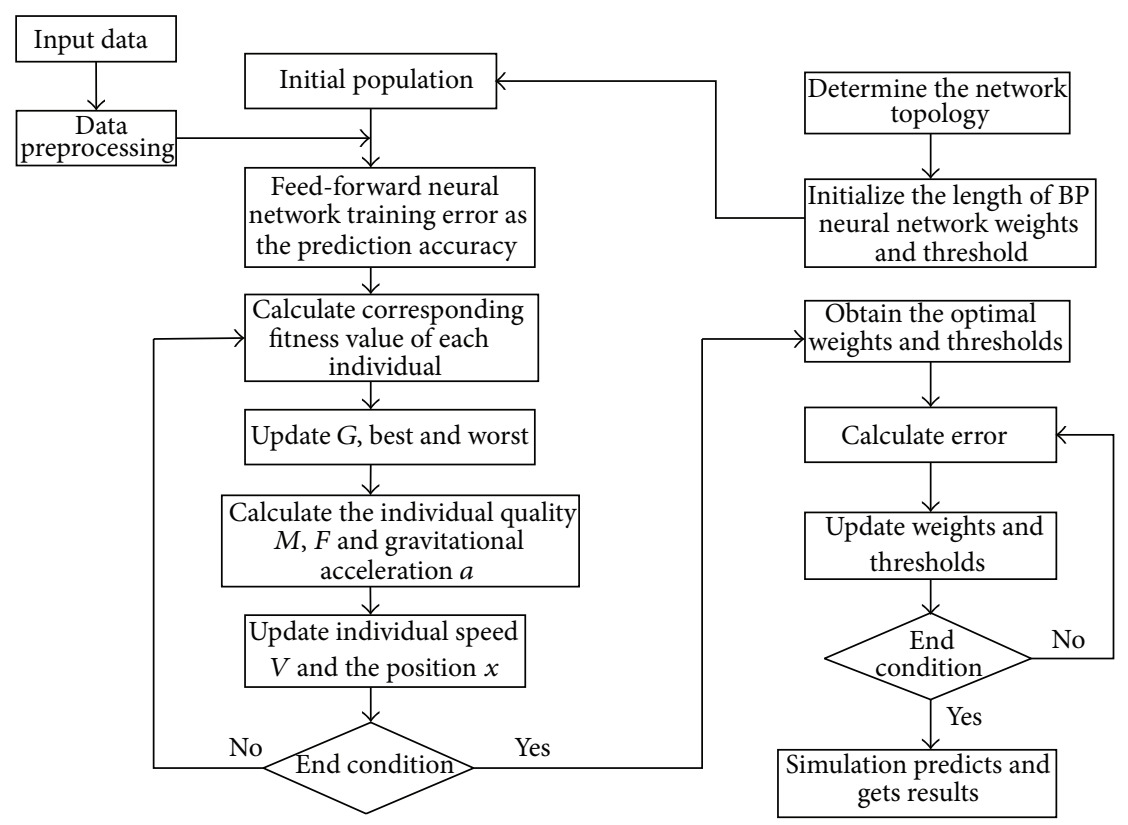

FIgURE 5: Flowchart of PSO-GSA optimizing FNN.

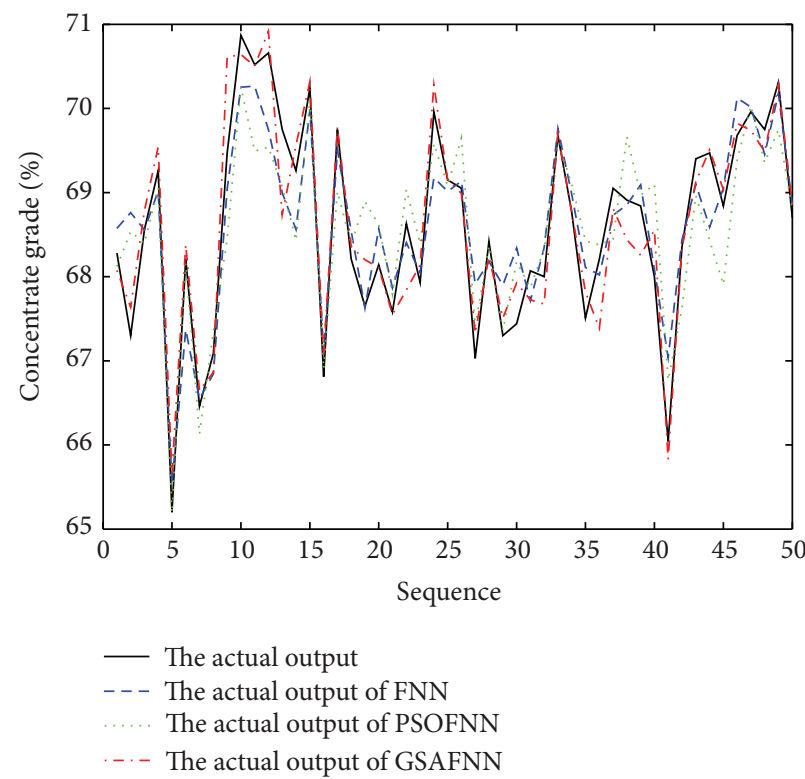

(a) Concentrate grade

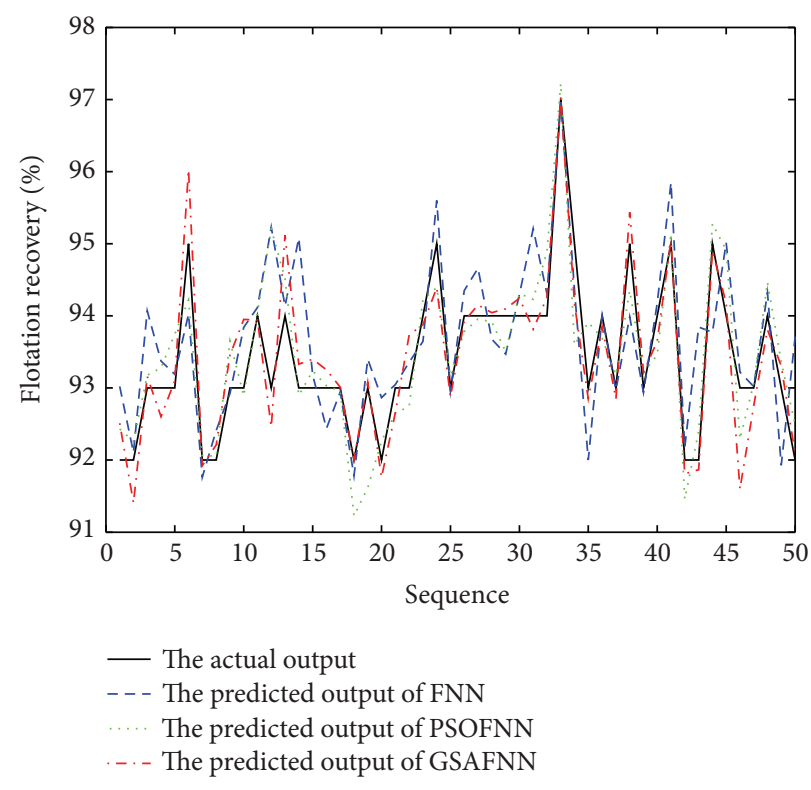

(b) Flotation recovery rate

FIGURE 6: Predicted results of soft-sensor models.

The activation function of FNN is tanh and the output uses the linear activation function. The initialization parameters of PSO-GSA algorithm are described as follows: $N=30$, $c_{1}=c_{2}=2, G_{0}=1$, inertia weight $w=2$, and the number of maximum iterations is $t=300$. Firstly, three soft-sensor models based on FNN, FNN optimized by PSO algorithm, and FNN optimized by GSA are established to realize the prediction of concentrate grade and recovery rate in the flotation process. Figure 6 is a comparison of the predicted output and the actual output under three models. Figure 7 is a comparison of the output prediction error curves under three models. It can be seen, from the predicted output curves and the prediction error curves, in these three models, that FNN soft-sensor models optimized by PSO algorithm and GSA have better predictive accuracy than the standard FNN soft-sensor model. Therefore, in order to verify the validity of PSO-GSA hybrid algorithm, the proposed PSO-GSA FNN soft-sensor model is compared with PSO-FNN model and 


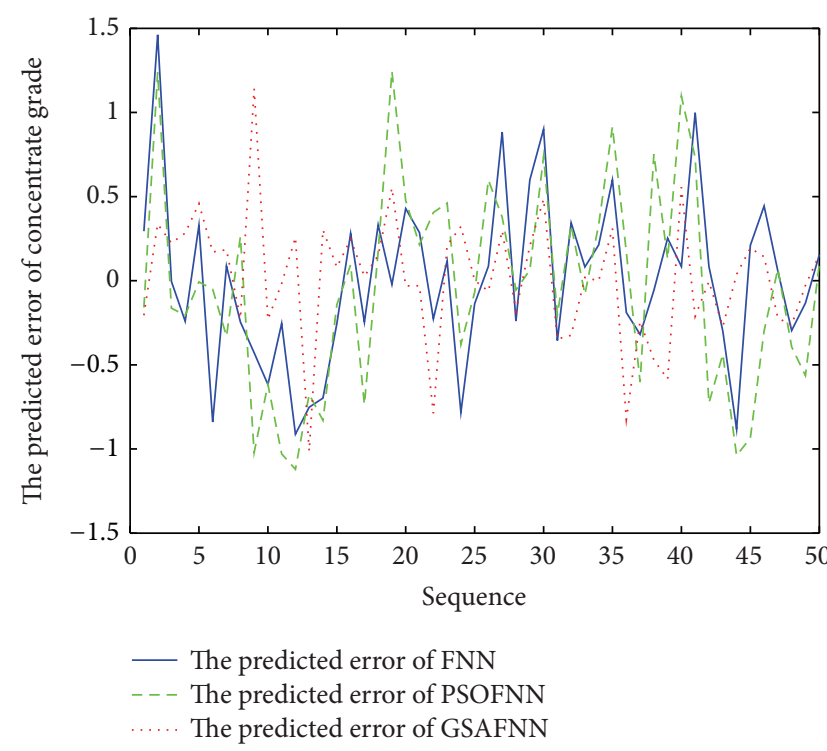

(a) Concentrate grade

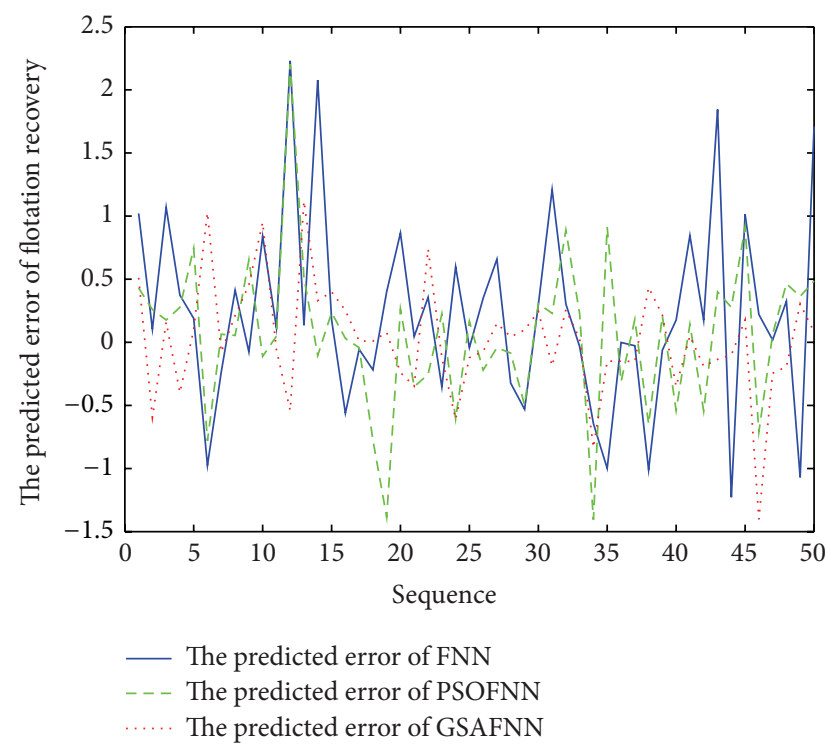

(b) Flotation recovery rate

FIGURE 7: Predicted errors of soft-sensor models.

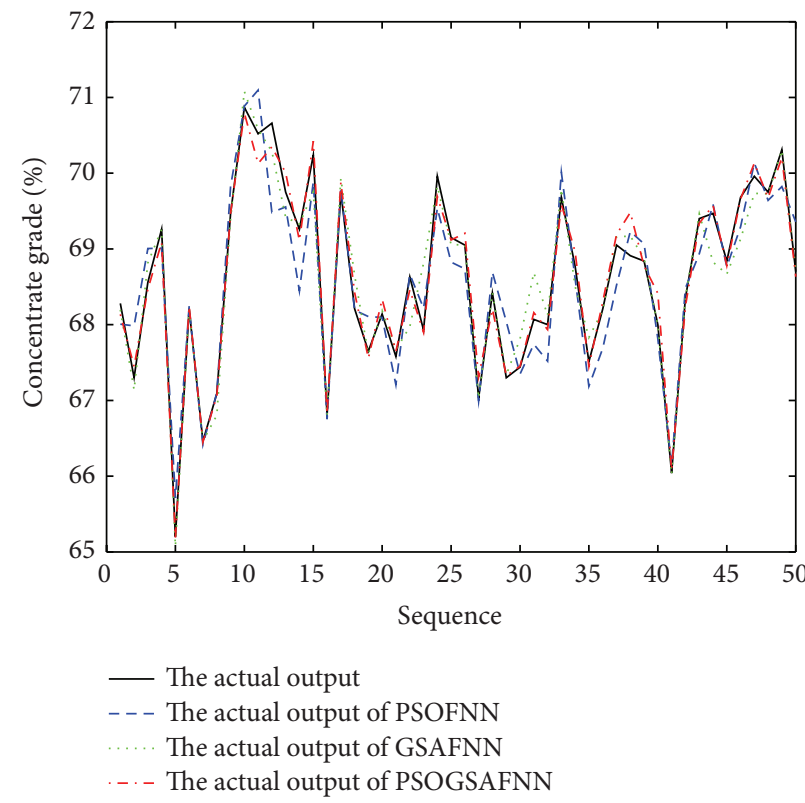

(a) Concentrate grade

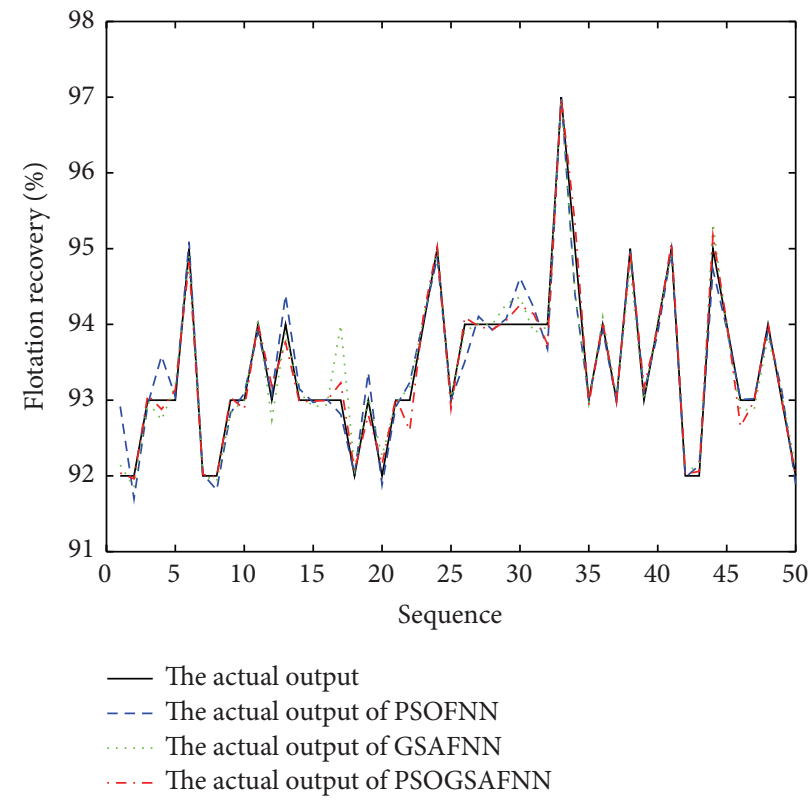

(b) Flotation recovery rate

FIGURE 8: Predicted results of soft-sensor models.

GSA-FNN model. Figure 8 is a comparison of the predicted output and the actual output under three models. Figure 9 is a comparison of the output prediction error curves under three models.

In order to compare the predictive ability and precision of these soft-sensor models based on the above-defined performance index, the performances are calculated and the results are shown in Table 2. Seen from Section 4, the prediction error of FNN soft-sensor model based on PSOGSA is minimum.

\section{Conclusion}

The five variables (feed grade, feed concentration, feed flow, agents flow, and feed granularity) are selected as the input variables of the discussed soft-sensor model. The flotation 


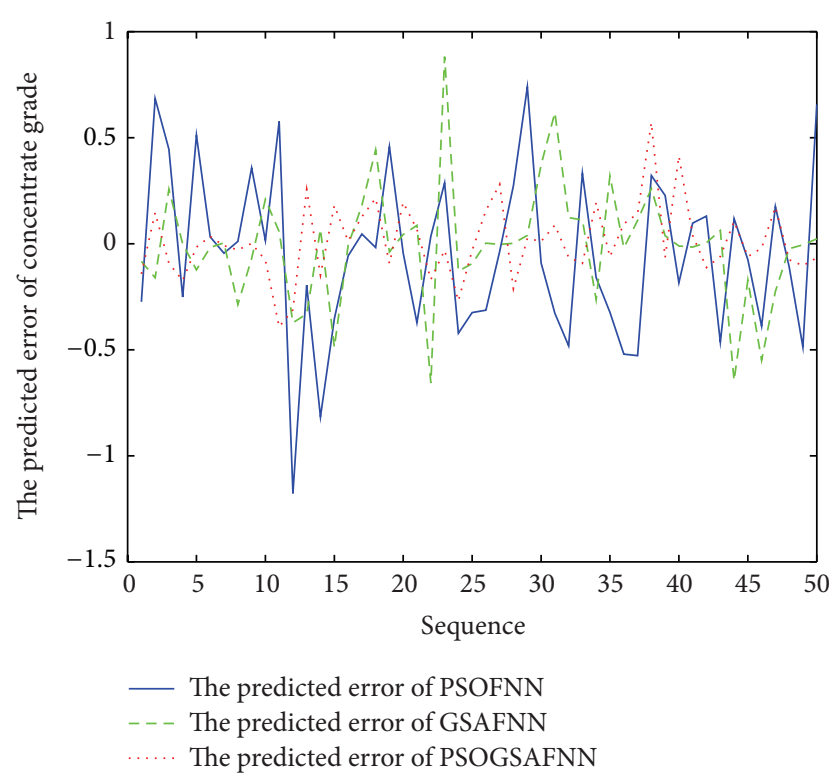

(a) Concentrate grade

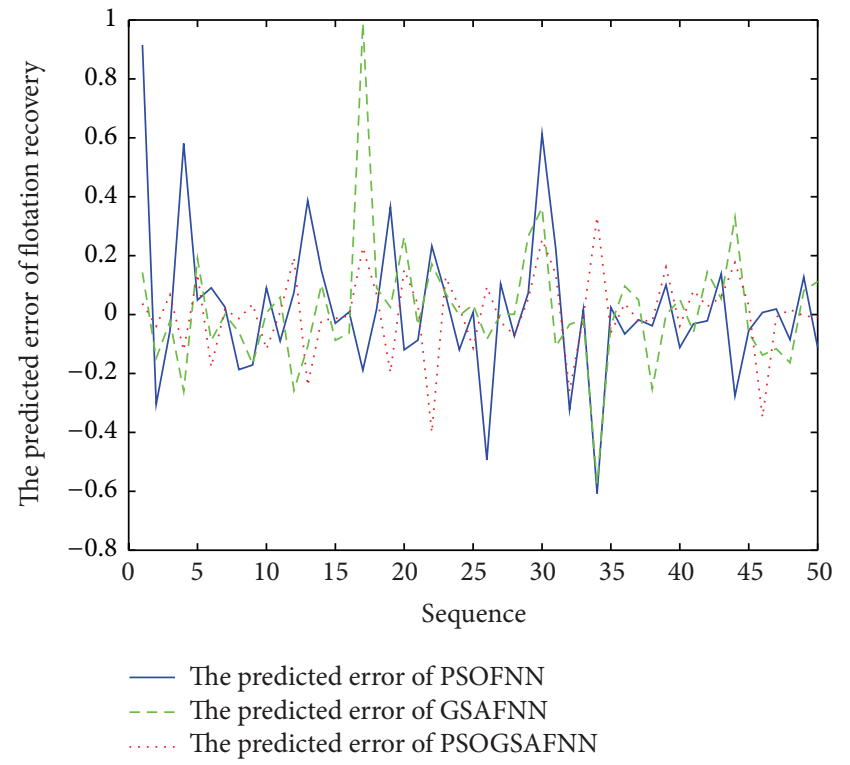

(b) Flotation recovery rate

FIGURE 9: The predicted error of soft-sensor model.

TABLE 2: Predictive performance comparison of soft-sensor models.

\begin{tabular}{lcccccc}
\hline Predictive object & Predictive method & MSE & RMSE & NRMSE & SSE & MAPE \\
\hline \multirow{2}{*}{ Concentrate grade (\%) } & FNN & 0.2416 & 0.4915 & 0.0223 & 12.081 & 0.5564 \\
& PSO-FNN & 0.1528 & 0.3909 & 0.0008 & 7.6402 & 0.4471 \\
& GSA-FNN & 0.0764 & 0.2764 & 0.0006 & 3.8209 & 0.2649 \\
& PSOGSA-FNN & 0.0300 & 0.1733 & 0.0004 & 1.5009 & 0.1890 \\
\hline \multirow{3}{*}{ Flotation recovery rate (\%) } & FNN & 0.1751 & 0.4184 & 0.0163 & 8.7535 & 0.3089 \\
& PSO-FNN & 0.0627 & 0.2505 & 0.0004 & 3.1372 & 0.1751 \\
& GSA-FNN & 0.0447 & 0.2114 & 0.0003 & 2.2341 & 0.1422 \\
& PSOGSA-FNN & 0.0194 & 0.1393 & 0.0002 & 0.9707 & 0.1054 \\
\hline
\end{tabular}

concentrate grade and recovery rate are output variables. The hybrid algorithm combining PSO algorithm and GSA is used to optimize the parameters of FNN soft-sensor model in order to improve the predictive accuracy. It can be seen, from the prediction results and comparison results, that the FNN soft-sensor model based on the proposed PSO-GSA algorithm has the highest prediction accuracy compared with the other soft-sensor models, which can meet the online softsensor requirements of the real-time control in the flotation process.

\section{Conflict of Interests}

The authors declare no conflict of interests.

\section{Authors' Contribution}

Jie-Sheng Wang participated in the concept, design, and interpretation and commented on the paper. Shuang Han participated in the data collection, analysis, algorithm simulation, the draft writing, and critical revision of this paper.

\section{Acknowledgments}

This work is partially supported by the Program for China Postdoctoral Science Foundation (Grant no. 20110491510), the Program for Liaoning Excellent Talents in University (Grant no. LR2014008), the Project by Liaoning Provincial Natural Science Foundation of China (Grant no. 2014020177), and the Program for Research Special Foundation of University of Science and Technology of Liaoning (Grant no. 2011ZX10).

\section{References}

[1] D. Hodouin, S.-L. Jämsä-Jounela, M. T. Carvalho, and L. Bergh, "State of the art and challenges in mineral processing control," Control Engineering Practice, vol. 9, no. 9, pp. 995-1005, 2001.

[2] J. Kaartinen, J. Hätönen, H. Hyötyniemi, and J. Miettunen, "Machine-vision-based control of zinc flotation-a case study," Control Engineering Practice, vol. 14, no. 12, pp. 1455-1466, 2006.

[3] B. J. Shean and J. J. Cilliers, "A review of froth flotation control," International Journal of Mineral Processing, vol. 100, no. 3-4, pp. 57-71, 2011. 
[4] J. M. Hargrave and S. T. Hall, "Diagnosis of concentrate grade and mass flowrate in tin flotation from colour and surface texture analysis," Minerals Engineering, vol. 10, no. 6, pp. 613621, 1997.

[5] G. Bartolacci, P. Pelletier Jr., J. Tessier Jr., C. Duchesne, P.-A. Bossé, and J. Fournier, "Application of numerical image analysis to process diagnosis and physical parameter measurement in mineral processes. Part I. Flotation control based on froth textural characteristics," Minerals Engineering, vol. 19, no. 6-8, pp. 734-747, 2006.

[6] S. H. Morar, M. C. Harris, and D. J. Bradshaw, "The use of machine vision to predict flotation performance," Minerals Engineering, vol. 36-38, pp. 31-36, 2012.

[7] D. W. Moolman, C. Aldrich, J. S. J. Van Deventer, and D. J. Bradshaw, "The interpretation of flotation froth surfaces by using digital image analysis and neural networks," Chemical Engineering Science, vol. 50, no. 22, pp. 3501-3513, 1995.

[8] C.-H. Yang, J.-Y. Yang, X.-M. Mou, K.-J. Zhou, and W.-H. Gui, "A segmentation method based on clustering pre-segmentation and high-low scale distance reconstruction for colour froth image," Journal of Electronics \& Information Technology, vol. 30, no. 6, pp. 1286-1290, 2008.

[9] J. Wang, Y. Zhang, and S. Sun, "Multiple T-S fuzzy neural networks soft sensing modeling of flotation process based on fuzzy C-means clustering algorithm," in Advances in Neural Network Research and Applications, vol. 67 of Lecture Notes in Electrical Engineering, pp. 137-144, Springer, Berlin, Germany, 2010.

[10] C.-H. Yang, H.-F. Ren, C.-H. Xu, and W.-H. Gui, "Soft sensor of key index for flotation process based on sparse multiple kernels least squares support vector machines," The Chinese Journal of Nonferrous Metals, vol. 21, no. 12, pp. 3149-3154, 2011.

[11] H. Li, T. Chai, and H. Yue, "Soft sensor of technical indices based on KPCA-ELM and application for flotation process," CIESC Journal, vol. 63, no. 9, pp. 2892-2898, 2012.

[12] K.-J. Zhou, C.-H. Yang, X.-M. Mou, and W.-H. Gui, "Intelligent prediction algorithm for floatation key parameters based on image features extraction," Control and Decision, vol. 24, no. 9, pp. 1300-1305, 2009.

[13] J.-S. Wang and Y. Zhang, "Application of the soft sensing model based on the adaptive network-based fuzzy inference system (ANFIS) to the flotation process," Journal of Hefei University of Technology, vol. 29, no. 11, pp. 1365-1369, 2006.

[14] Z.-X. Geng and T.-Y. Chai, "Soft sensor of technical indices based on LS-SVM for flotation process," Journal of System Simulation, vol. 20, no. 23, pp. 6321-6324, 2008.

[15] J. Wang, S. Han, N. Shen, and S. Li, "Features extraction of flotation froth images and BP neural network soft-sensor model of concentrate grade optimized by shuffled cuckoo searching algorithm," The Scientific World Journal, vol. 2014, Article ID 208094, 17 pages, 2014.

[16] J.-S. Wang, S. Han, and N.-N. Shen, "Improved GSO optimized ESN soft-sensor model of flotation process based on multisource heterogeneous information fusion," The Scientific World Journal, vol. 2014, Article ID 262368, 12 pages, 2014.

[17] S.-Y. Ho, H.-S. Lin, W.-H. Liauh, and S.-J. Ho, "OPSO: orthogonal particle swarm optimization and its application to task assignment problems," IEEE Transactions on Systems, Man and Cybernetics, Part A: Systems and Humans, vol. 38, no. 2, pp. 288298, 2008.

[18] R. Coban, "A fuzzy controller design for nuclear research reactors using the particle swarm optimization algorithm,"
Nuclear Engineering and Design, vol. 241, no. 5, pp. 1899-1908, 2011.

[19] E. Rashedi, H. Nezamabadi-pour, and S. Saryazdi, "GSA: a gravitational search algorithm," Information Sciences, vol. 179, no. 13, pp. 2232-2248, 2009. 

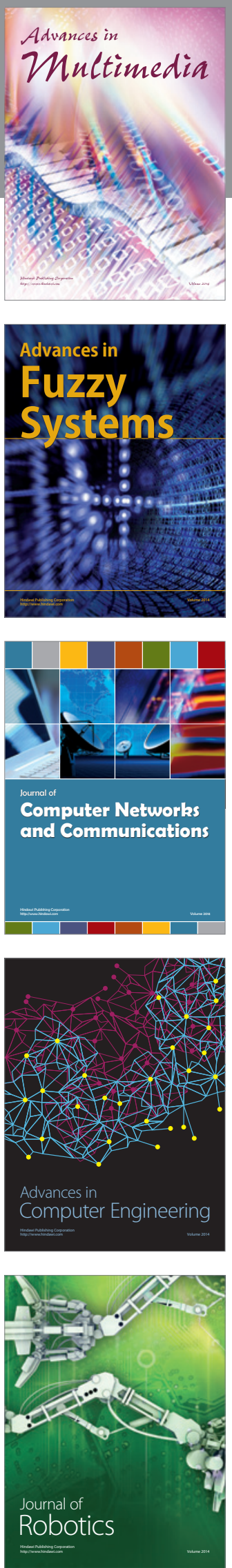

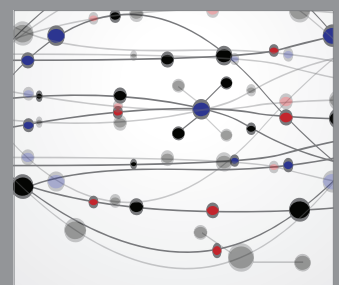

The Scientific World Journal
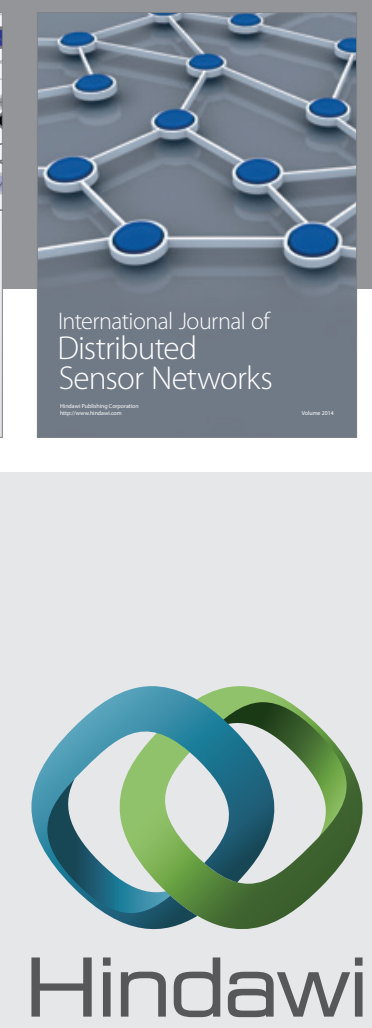

Submit your manuscripts at

http://www.hindawi.com
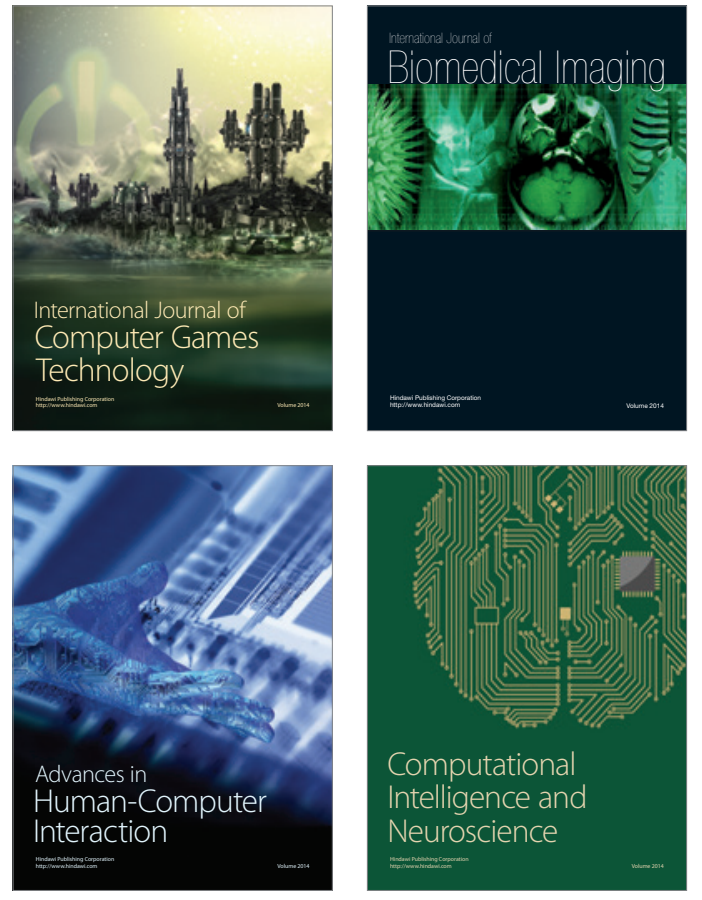
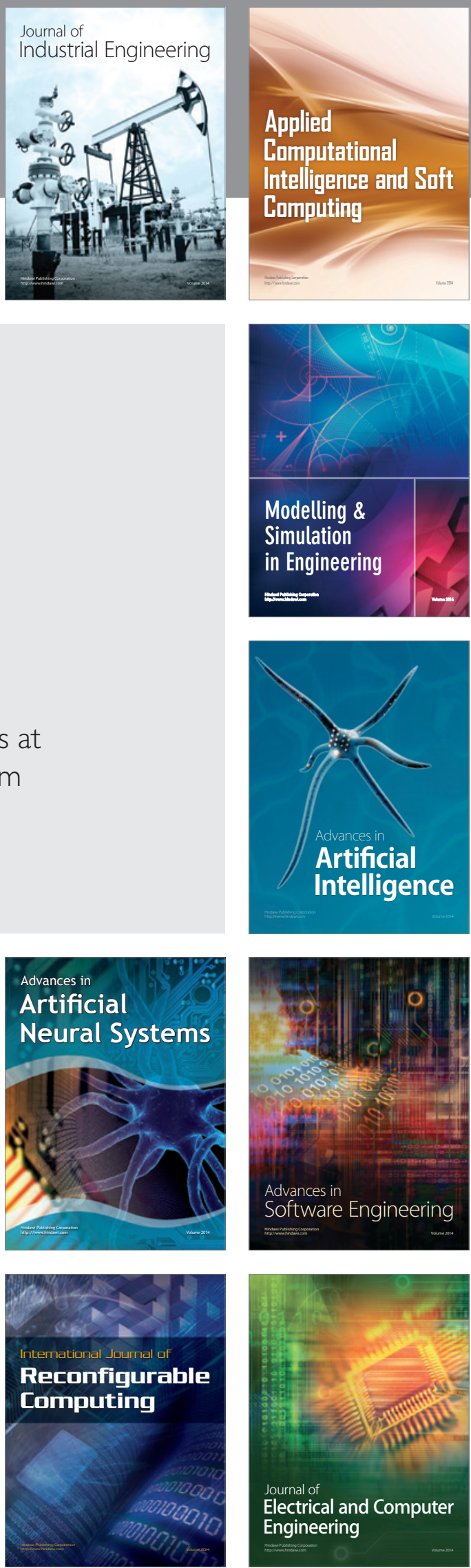